\title{
The ex vivo human lung: research value for translational science
}

\author{
James T. Ross, ${ }^{1}$ Nicolas Nesseler, ${ }^{2,3,4,5}$ Jae-Woo Lee, ${ }^{6}$ Lorraine B. Ware, ${ }^{7}$ and Michael A. Matthay ${ }^{6,8}$ \\ 1'Department of Surgery and ${ }^{2}$ Cardiovascular Research Institute, UCSF, San Francisco, California, USA. ${ }^{3}$ Department of \\ Anesthesia and Critical Care, Pontchaillou, University Hospital of Rennes, Rennes, France. ${ }^{4}$ Univ Rennes, CHU de Rennes, \\ Inra, INSERM, Institut Nutrition, Métabolismes, Cancer- UMR_A 1341, UMR_S 1241, Rennes, France. ' Univ Rennes, CHU \\ Rennes, INSERM, Centre d'Investigation Clinique de Rennes 1414, Rennes, France. 'Department of Anesthesiology, \\ Cardiovascular Research Institute, UCSF, San Francisco, California. 'Division of Allergy, Pulmonary and Critical Care \\ Medicine, Department of Medicine, and Department of Pathology, Microbiology and Immunology, Vanderbilt University \\ School of Medicine, Nashville, Tennessee, USA. ${ }^{8}$ Department of Medicine, Cardiovascular Research Institute, UCSF, San \\ Francisco, California, USA.
}

Respiratory diseases are among the leading causes of death and disability worldwide. However, the pathogenesis of both acute and chronic lung diseases remains incompletely understood. As a result, therapeutic options for important clinical problems, including acute respiratory distress syndrome and chronic obstructive pulmonary disease, are limited. Research efforts have been held back in part by the difficulty of modeling lung injury in animals. Donor human lungs that have been rejected for transplantation offer a valuable alternative for understanding these diseases. In 2007, our group developed a simple preparation of an ex vivo-perfused single human lung. In this Review, we discuss the availability of donor human lungs for research, describe the ex vivo-perfused lung preparation, and highlight how this preparation can be used to study the mechanisms of lung injury, to isolate primary cells, and to test novel therapeutics.

Authorship note: JTR and NN contributed equally to this work.

Conflict of interest: MAM is a principal investigator for an observational research study of pulmonary hypertension in patients with acute respiratory distress syndrome funded by Bayer Pharmaceuticals. LBW serves as a consultant for CSL Behring, Quark Pharmaceuticals, and Bayer Pharmaceuticals.

Copyright: (c) 2019 American Society for Clinical Investigation

Reference information: /CI Insight. 2019;4(11):e128833. https://doi. org/10.1172/ji.insight.128833.

\section{Introduction}

Animal models and experiments using primary lung tissue or cells from animals have formed the basis for much of our understanding of human pulmonary physiology and pathophysiology. However, current animal models of pulmonary disease are limited by differences in the basic pulmonary anatomy and physiology between animals and humans, an inability to faithfully model human pathology, and the genetic and environmental homogeneity of laboratory-bred animals (1). Clinical trials also present significant challenges because trials are time-consuming and expensive, interventions are limited, and human tissues are not routinely available (2). Donor human lungs have become a valuable resource to carry out studies of human pulmonary disease because of their broad genetic and environmental heterogeneity.

Ex vivo human lung studies offer a useful additional experimental preparation to animal models, and substantial contributions have already been made with nonperfused and perfused ex vivo preparations and with cells isolated from the human lung. Early studies with nonperfused human lungs, for example, demonstrated that treatment with a $\beta$-adrenergic agonist markedly increases alveolar fluid clearance (AFC) (3) and highlighted the high quality of many donor lungs that were rejected for transplantation (4). Systems for the ex vivo perfusion of a pair of lungs (EVLP) were developed in the 1950s, though the first major clinical trial of ex vivo perfusion before lung transplantation was not performed until 2011. In a landmark trial, high-risk donor lungs that remained stable with EVLP for 4 hours before transplantation had clinical outcomes similar to those of usual-risk, conventionally selected lungs (5). Since this initial success, EVLP has proved an important tool for assessment, preservation, and conditioning of donor lungs before transplantation (6).

In 2007, our group developed a simple and inexpensive noncommercial system for the ex vivo perfusion of single human lungs (7). For the last 11 years, this ex vivo-perfused human lung has been used to study the pathophysiology of acute lung injury and to test new therapeutics. The ex vivo lung preparation is a convenient resource to study the whole human lung in the absence of the liver, spleen, or other organs. In addition to the scientific value of the isolated perfused lung, donor lungs have also 
proved a valuable source of primary human cells, including alveolar epithelial type II cells, fibroblasts, and macrophages. Pathways or targets identified through in vitro systems or early animal models can be investigated in human tissue before committing to the complexity, time, and cost of human trials. These studies form part of a new paradigm for translational science.

This Review will discuss the practical issues in obtaining human lungs for research, provide an overview of preparations of ex vivo-perfused human lungs, and highlight selected research contributions that have been made using this model. The focus will be on the use of the ex vivo human lung system to understand the pathophysiology of acute and chronic lung injury, the value of cellular studies using the human lung, and the testing of therapeutics in the ex vivo lung.

\section{How to obtain human lungs for research}

On average, approximately 2300 lung transplants are performed in the United States each year, but, due in part to the relatively restrictive criteria that govern organ selection in lung transplants, this represents only $24 \%$ of available donor lungs (8). Our studies have reported that most of the lungs that are not transplanted are in good condition, with no or mild pulmonary edema, intact basal AFC, and normal or only mildly abnormal histologic findings $(4,7)$. These human lungs are potentially available for research via agreement with 1 of the 58 regional organ procurement organizations (OPOs) in the United States.

The use of donor human lungs for research requires explicit permission from the donor's family, which is solicited by the OPO as part of the standard organ donation process. Local institutional review board approval is not required because research on tissues from deceased organ donors is not considered human subject research, though appropriate institutional biosafety approvals should be sought for handling human tissue. Once a relationship with an OPO has been established, a network can be built within the receiving institution to allow each pair of donor lungs to be used by multiple research groups. Typically, a procurement and transportation fee is paid to the OPO. For example, at UCSF, donor lungs are delivered by the OPO to the UCSF Medical Center operating room. When releasing a pair of lungs for research, the OPO sends an email to a full list of the research laboratories involved at UCSF. An investigator from the UCSF group evaluates the lungs and the clinical history of the donor, and whole lungs, individual lobes, or samples of the parenchyma or airways are distributed to investigators from several teams. Thus, almost every portion of the lungs, which would otherwise have been discarded, can be used for research.

\section{Available clinical data}

Human lungs rejected for transplantation offer investigators the combination of an intact human organ and extensive clinical data. The deidentified donor data that are routinely available include the mode of donation (brain-dead donor, donor after cardiac death); cause of death; medical, surgical, and tobacco use history; and results of recent imaging, including chest radiographs, ventilator parameters, and arterial blood gases. These comprehensive data offer an important opportunity to address pressing clinical questions.

For example, a scoring system for the extent of pulmonary edema was developed based on the routine pre-procurement chest radiograph. The score strongly correlated with the total excised lung weight, indicating that pulmonary edema in a potential donor could be semiquantified using the chest radiograph (9). A refined scoring system, the Radiographic Assessment of Lung Edema, was developed in a second cohort of lung donors and was strongly associated with disease severity and mortality in a separate cohort of patients with acute respiratory distress syndrome (ARDS) (10). In subsequent work, human lungs were evaluated to study the mechanisms by which donor smoking leads to poor lung transplant outcomes (11). In this study, lungs were recovered from 298 donors and compared with lungs from never smokers. The lungs of current or past smokers had higher recovered lung weights, suggestive of more pulmonary edema. Donors with smoking histories of 20 or more pack-years had lower rates of AFC, a change also found in lung transplant recipients with primary graft dysfunction (12). In addition, current or ever smokers had higher levels of the proinflammatory cytokine IL-8 in the alveolar fluid and decreased levels of surfactant protein D, a change that is associated with lung epithelial injury. Together, these findings indicated that donor smoking is associated with alveolar epithelial dysfunction and that release of proinflammatory mediators might contribute to pulmonary edema in the lung before transplant and to primary graft dysfunction after transplant. 


\section{Isolation of cells from human lungs}

Human donor lungs have proved an excellent source of primary human cells, including alveolar epithelial type II (ATII) cells, airway epithelial cells, fibroblasts, endothelial cells, and macrophages. Modern molecular biology techniques have been applied to characterize these populations with considerable success, and the use of primary cells from human donor lungs has provided insight into the pathophysiology of both acute lung diseases, such as pneumonia and ARDS, and chronic diseases, such as chronic obstructive pulmonary disease and cystic fibrosis.

Isolated ATII cells provided the basis for understanding the mechanisms of vectorial ion transport across the pulmonary epithelium. Human ATII cells are collected by enzymatic digestion of the lung tissue, and the filtered cells are seeded on collagen-coated Transwell plates (Figure 1). Cells typically reach confluence at 48 hours, and after 72 hours, the fluid in the top compartment is removed to allow cells to grow at the air-fluid interface $(13,14)$. Yields are typically greater than $2.0 \times 10^{6}$ cells per gram of lung tissue, with $90 \%( \pm 3 \%)$ purity at the time of isolation. Electron microscopy demonstrates that ATII cells grown in monolayers retain characteristic ultrastructural features of ATII cells, including a cuboidal shape, lamellar bodies, and microvilli (15). This model has been used to show that the cystic fibrosis transmembrane conductance regulator (CFTR) is functionally expressed in human ATII cells (15). CFTR, the luminal epithelial $\mathrm{Na}^{+}$channel $(\mathrm{ENaC})$, and basolateral $\mathrm{Na}^{+} / \mathrm{K}^{+}$-ATPase were also shown to be required for cAMP-regulated ion and fluid transport across human ATII cells. Recently, another group reported a potential protective role for rare mutations in the $\delta$ - $\mathrm{ENaC}$ subunit in cystic fibrosis that associated with a nonprogressive phenotype (16). The authors of this study isolated epithelial cells $\left(\mathrm{CD} 45^{-} \mathrm{CD} 31^{-} \mathrm{epCAM}^{+}\right)$ from human donor lungs and demonstrated that the $\delta$-ENaC-encoding gene (SCNN1D) is expressed primarily in alveolar epithelial cells that express all the subunits of $\mathrm{ENaC}$ that are critical for AFC.

This ATII monolayer model has also provided important insights into the mechanisms of impaired AFC, which is known to be associated with poor clinical outcomes, in acute lung injury (17). For example, ATII cell monolayers incubated with pulmonary edema fluid from patients with sepsis-associated ARDS had a reduction in net vectorial fluid transport and an increase in paracellular protein permeability compared with monolayers incubated with simultaneously collected plasma (18). Impaired vectorial fluid transport associated with reduced expression of all the major sodium and chloride transporters at the gene and protein level, and the decrease in these transporters was responsible, in part, for impaired AFC in ARDS. Further work with ATII monolayers also identified the mechanisms by which bone marrowderived human mesenchymal stem cells (MSCs, also referred to as mesenchymal stromal cells) could therapeutically reverse these types of inflammatory injury. Coculture of MSCs with an ATII monolayer restored net fluid transport and reduced epithelial protein permeability following an inflammatory insult, in part via paracrine factors, including angiopoietin-1, lipoxin A4, and keratinocyte growth factor (19-21). Gene expression analysis of cultured human ATII cells indicated that human MSCs also partially reverse the upregulation of inflammatory genes and downregulation of genes associated with surfactant production and AFC that occur in response to proinflammatory cytokine stimulation (22).

Donor human lungs have also been used as a source of mesenchymal cells, including fibroblasts. For example, one research group isolated mesenchymal cells from the airways of donor human lungs and used single-cell RNA sequencing to demonstrate that these cells have an identity that segregates depending on where in the lung they are isolated. Hedgehog was preferentially expressed in mesenchymal cells isolated from the proximal airway compared with those isolated from the distal airway, and this gradient of hedgehog expression associated with distinct mesenchymal gene signatures (23). Disruption of this gradient via overexpression of a hedgehog effector throughout the airway in a mouse model resulted in a proximal airway-like phenotype, including loss of alveoli and airspace enlargement, in the distal airways. These studies offer insight into the pathogenesis of emphysema because GWAS have mapped susceptibility loci for emphysema to genes that alter hedgehog signaling.

Other investigators have isolated and cultured fibroblasts from donor human lungs to study cigarette smoke-induced inflammation and remodeling $(24,25)$. Application of cigarette smoke extract to primary human pulmonary fibroblasts induced expression of the transcription factor early growth response 1 , thereby promoting synthesis and secretion of matrix metalloproteinase-2 and HSP70. Secreted HSP70 acted in a paracellular manner to stimulate the release of IL-8, a potent neutrophil chemoattractant, from other pulmonary fibroblasts. Together these studies demonstrated an important pathway by which cigarette smoke exposure may lead to emphysema.

Donor human lungs have also been used to study the distribution and function of pulmonary immune cells. Another research group recently analyzed the pulmonary mononuclear phagocyte sys- 

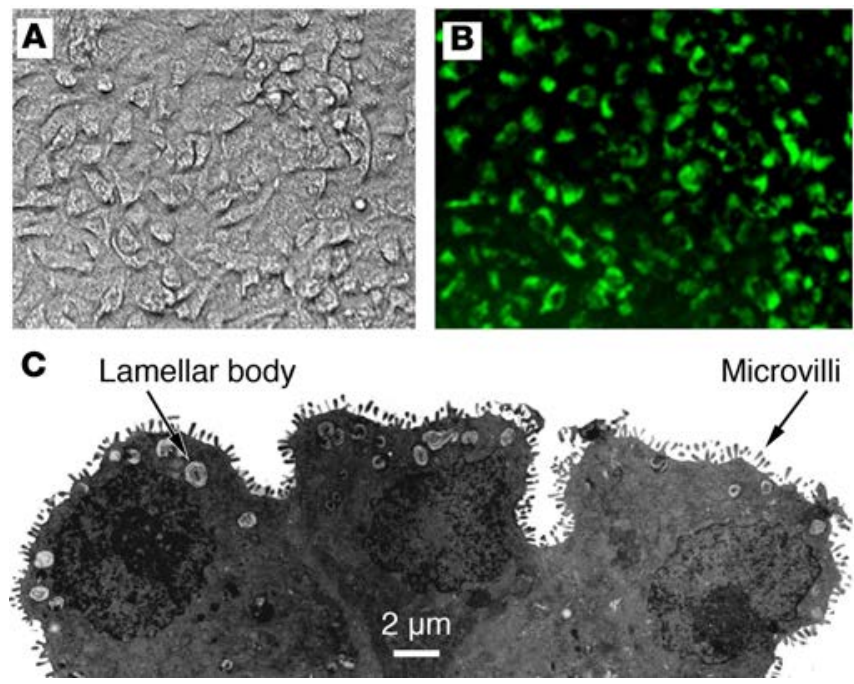

Figure 1. Primary human ATII cells grown on a collagen I-coated Transwell membrane. (A) Phase contrast image of an ATII monolayer. (B) An ATII monolayer stained with LysoTracker DND-26 green DDND-26 (Molecular Probes) to selectively stain lamellar bodies. (C) Electron microscopy of the side view of human ATII cells to demonstrate lamellar bodies and microvilli. Figure adapted from Fang et al. (15) and used with permission.

tem of lung and the lung-draining lymph nodes from 72 human donors (26). The investigators used intrabronchial and intravenous antibody labels to differentiate tissue-resident mononuclear phagocytes from cells within the vasculature, digested the lung tissue, and used flow cytometry to characterize 5 populations of resident mononuclear cells. These data are of interest because the majority of our prior knowledge of the pulmonary mononuclear phagocyte system was based on animal studies or samples from tumor resection margins and not from healthy whole human organs. Finally, donor human lungs have proved a useful source of pulmonary vascular cells. Pulmonary arterial endothelial cells, endothelial cells of the microvasculature, and pulmonary arterial smooth muscle cells have all been isolated and cultured from human donor lungs (27).

In summary, donor human lungs have proved a valuable source of primary human pulmonary cells, which have contributed to our understanding of pulmonary physiology and immunology and of the pathophysiology of acute lung injury, emphysema, and cigarette smoke-associated lung diseases. Importantly, these cells may be isolated from previously healthy donors or from those affected by the pathologic process of interest. This is an important difference from other sources of primary human pulmonary cells, which are often isolated from biopsy or pulmonary resection specimens. An important future target will be to successfully culture alveolar type I (ATI) cells.

\section{Ex vivo-perfused human lung}

All the authors of this Review currently carry out experiments with the ex vivo-perfused human lung, each with minor variations and refinements. Here we describe the core principles of the preparation and discuss the major differences and decision points in the model setup.

Lung selection. Human lungs that have been rejected for transplantation are received via agreement with the local OPO. The majority of lungs are retrieved from brain-dead donors, and lungs from donors after cardiac death have not been rigorously evaluated in the ex vivo-perfused lung model. At procurement, the vascular compartment is flushed with low-potassium dextran solution at $4^{\circ} \mathrm{C}$, lungs are inflated to peak pressure of $20 \mathrm{cmH}_{2} \mathrm{O}$ with $\mathrm{FiO}_{2}$ of 0.5 , and the trachea is clamped. Lungs are transported at $4^{\circ} \mathrm{C}$ and are not perfused during transport. All the authors use lungs with a total ischemia time of under 48 hours.

Lungs with gross rupture of the visceral pleura or major vascular injury cannot be reliably perfused, though minor bronchial injuries can be repaired. An approximately $1-\mathrm{cm}$ length of pulmonary artery proximal to the first arterial branch point is required to ensure reliable arterial cannulation. Beyond this, the degree of organ selection should be based on the research question. Quality metrics have included using only lungs with a baseline AFC rate within a certain range ( $>10 \% / \mathrm{h}$, explained below) (28) or only lungs that reach a stable weight within 30-60 minutes after rewarming (29). For most experiments, the right and left lungs may be considered interchangeable. Depending on the hypothesis to be tested, a single lung may serve as its own control (30), 2 lobes of a single lung may serve as intervention and control (7), or the right and left lung may be perfused separately as intervention and control (29). 
Preparation. The right and left lungs are separated and 1 lung selected. A cannula is placed in the pulmonary artery and secured with a purse-string suture. The main bronchus is cannulated with an approximately $15-\mathrm{cm}$ length of standard 7.5 endotracheal tube, which is secured via a suture placed through one wall of the cartilaginous trachea. The lung preparation is weighed and suspended from a mass transducer (Harvard Apparatus) in a custom-made acrylic chamber. The chamber is partially submerged in a water bath, and the base of the chamber serves as a reservoir for the perfusate solution. The temperature of the perfusate at the pulmonary vein is maintained at $37^{\circ} \mathrm{C}$.

Perfusion. The lung is perfused with 2 liters of DME-H21 high-glucose medium without phenol red containing $5 \%$ BSA and warmed to $37^{\circ} \mathrm{C}$. The lung preparation (Figure 2) is perfused with a roller pump (Terumo Sarns) via a cannula secured in the pulmonary artery. The pulmonary veins are not cannulated; thus, the perfusate drains passively from the pulmonary veins into the reservoir at the base of the acrylic chamber, where it is recycled through the pump. The pulmonary arterial pressure is measured via a pulmonary arterial catheter (Cook) placed in the circuit at approximately the level of the left atrium. The pump flow rate (usually about $0.2 \mathrm{l} / \mathrm{min}$ ) is adjusted to maintain a pulmonary arterial pressure of 10 to $12 \mathrm{mmHg}$ (Biopac) to prevent hydrostatic pulmonary edema.

In early experiments, investigators discovered that the addition of fresh whole human blood to the perfusate was required to generate a robust inflammatory response to $E$. coli endotoxin (21). Subsequent experiments were conducted with $(29,31)$ or without $(32)$ the addition of $100 \mathrm{ml}$ of fresh human blood to the perfusate, depending on the relevance of the inflammatory response to the experimental design. Type-specific blood is not required because the exposure is brief and the majority of circulating leukocytes in the lungs will have been removed in the procurement process.

Ventilation and oxygenation. Studies with the ex vivo-perfused lung preparation can be done with CPAP of 8 or $10 \mathrm{cmH}_{2} \mathrm{O}(7,11,21,28,33)$, and recruitment maneuvers to $25 \mathrm{cmH}_{2} \mathrm{O}$ may be added at the start of the experiment (29). Two studies used positive-pressure ventilation (tidal volume $300 \mathrm{ml}$, respiratory rate $10 / \mathrm{min}, \mathrm{FiO}_{2}$ 0.21) $(30,32)$. To date, the majority of studies have been performed with $95 \% \mathrm{O}_{2}$ and $5 \%$ $\mathrm{CO}_{2}(11,21,28,31,33)$, though 3 studies have been performed with room air $\left(\mathrm{FiO}_{2}\right.$ of 0.21$)(29,30,32)$.

The impact of selecting CPAP versus mechanical ventilation and of the addition of recruitment maneuvers has not been directly evaluated. However, several groups have reported adequate oxygenation levels independent of the fractional concentration of oxygen or the ventilation method used, perhaps because of minimal metabolic demand of the isolated perfused human lung. For example, one group reported $\mathrm{PaO}_{2}$ of 130-165 mmHg using a mechanical ventilator with positive end-expiratory pressure of $5 \mathrm{cmH}_{2} 0$ and $\mathrm{FiO}_{2}$ of 0.21 (32). Another group reported $\mathrm{PaO}_{2} / \mathrm{FiO}_{2}$ ratios of $188 \pm 20$ using $\mathrm{CPAP}$ with $\mathrm{FiO}_{2}$ of 0.95 (33). Of note, hyperoxia can be detrimental in the presence of cell-free hemoglobin (29).

\section{Experimental endpoints}

Acute lung injury involves the loss of endothelial and epithelial barrier functions, with increased movement of protein into the airspaces and decreased AFC (34). These findings are common to acute lung injury of many etiologies, including bacterial pneumonia and reperfusion injury after lung transplant (35). The ex vivo-perfused human lung preparation allows direct measurement of a range of physiologic parameters (Table 1) as well as histologic, microbiologic, and immunologic endpoints (Table 2). Here we highlight several important experimental endpoints that have been used across a range of injury models.

Lung weight gain and lung fluid balance. The ex vivo-perfused lung gains weight during perfusion through fluid accumulation in the interstitium and airspaces. Weight gain is therefore a good proxy for pulmonary edema formation. Following equilibration of the lung on the circuit, net weight gain represents a balance of fluid movement across the endothelium into the interstitium and across the epithelium into the airspaces. In vivo, fluid is also removed from the interstitium via lymphatic drainage and transpleural leakage (36). However, for this preparation, we assume that fluid loss via both routes is minimal. In addition to Starling forces, 2 other factors affect the accumulation of pulmonary edema. First, the capacity of the interstitium is finite (37), so fluid in excess of this limit must return to the vascular compartment or flood into the airspaces (34). Second, in addition to the hydrostatic and protein osmotic forces, alveolar edema fluid clearance depends on sodium transport-dependent mechanisms generated by both ATI and ATII cells (AFC, see below) (38).

Therefore, lung weight gain is proportional to the permeability of the endothelium and epithelium and inversely proportional to AFC rate (7). Lung weight can be measured by mass balance at the beginning and end of the experiment or continuously with the lung suspended from a mass transducer (29). 

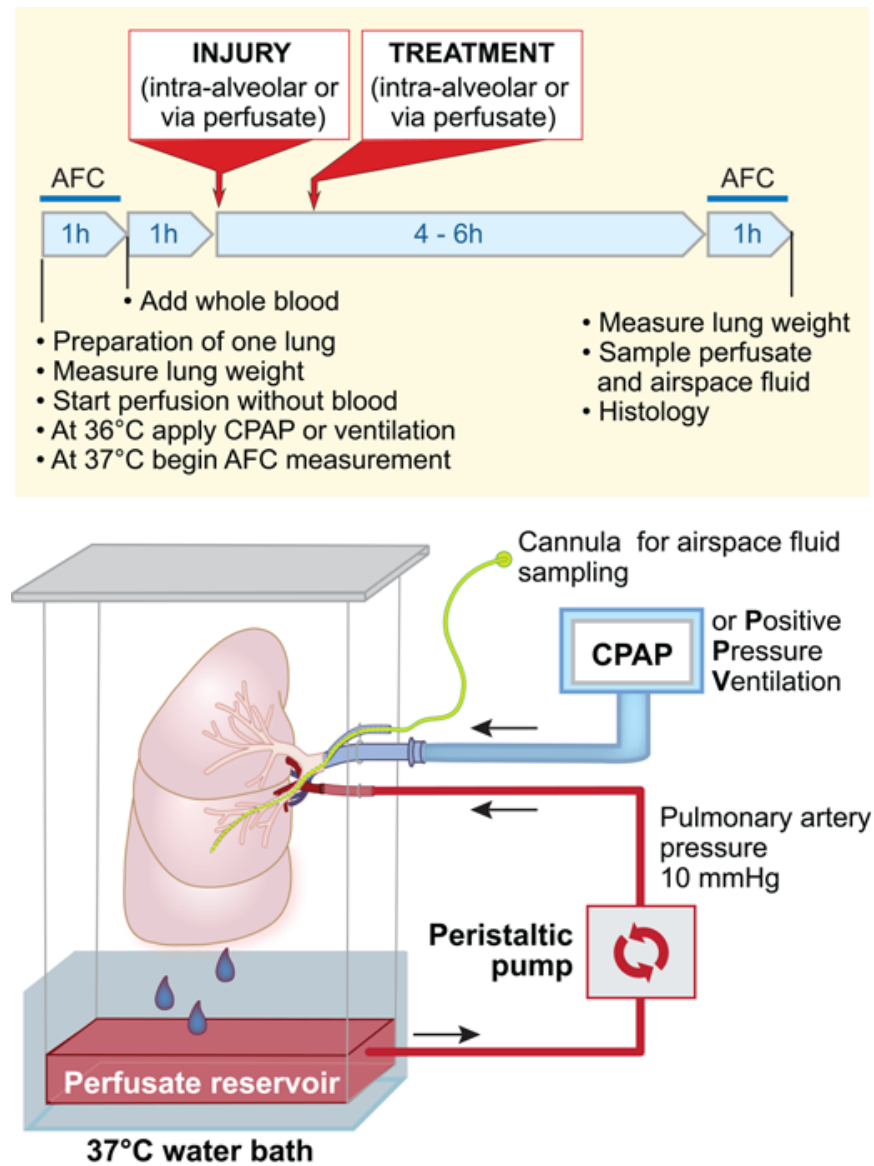

Figure 2. Schematic of the ex vivoperfused human lung preparation with experimental timeline. Human donor lungs rejected for transplantation are perfused with $5 \%$ BSA in DME-H21 high-glucose medium at $37^{\circ} \mathrm{C}$ via a cannula sewn into the pulmonary artery. The lung is inflated when the temperature reaches $36^{\circ} \mathrm{C}$, using continuous positive airway pressure (CPAP) or positive-pressure ventilation, with room air or a mix of $95 \% \mathrm{O}_{2}$ and $5 \% \mathrm{CO}_{2}$. Inflation at temperatures below $36^{\circ} \mathrm{C}$ produces lung injury (our unpublished observations). Then, 100 $\mathrm{ml}$ of fresh whole blood is added to the perfusate. The perfusion rate is adjusted to approximately $0.2 \mathrm{l} / \mathrm{min}$, via a roller pump, to maintain a pulmonary arterial pressure of $10 \mathrm{mmHg}$. Perfusate drains passively from the pulmonary veins into a reservoir at the bottom of the perfusion chamber. The main bronchus is cannulated with a standard endotracheal tube, and the lung is either inflated with CPAP or ventilated. A second cannula is inserted through a side port in the endobronchial tube and passed into the distal airspaces of the desired lobe, to allow sampling of the alveolar fluid. Following equilibration, the lung may be injured via the airspaces or perfusate, and treatment may be administered via the same routes.

Endothelial and epithelial permeability to protein. Acute lung injury is characterized by increased protein permeability across both the endothelium and epithelium. As a result, measures of protein permeability have been based on the movement of intravascular Evans blue-labeled albumin or exogenously added ${ }^{131}$ I-labeled albumin into the airspace fluid $(7,29)$ or into the extravascular compartment $(30)$. For example, one group (29) measured the movement of Evans blue-labeled albumin added to the perfusate across the endothelium and epithelium into the distal airspaces (29). The authors found that permeability to the labeled albumin was increased after injury with cell-free hemoglobin. The contribution of lung endothelial permeability was then studied by measuring the change in electrical impedance across a monolayer of cultured endothelial cells treated with cell-free hemoglobin. Another group studied the movement of Evans blue-labeled albumin into the extravascular compartment of lungs injured with intrabronchial E. coli bacteria (30). After a period of equilibration, Evans blue dye was added directly to the perfusate. Then, 90 minutes later, a sample of the perfusate was obtained, and the vascular compartment of the lung was flushed with phosphate-buffered saline. Sections from control and injured lobes were incubated with formamide to release the dye, and then permeability was estimated as a ratio of Evans blue in the injured and uninjured lobes.

$A F C$. Fluid is removed from the distal airspaces by an active ion transport process. In the alveolar epithelium, basolateral $\mathrm{Na}^{+} / \mathrm{K}^{+}$-ATPase pumps and apical ENaCs create an osmotic gradient that drives water reabsorption from alveolar edema fluid (34). AFC is increased in a cAMP-dependent fashion by $\beta_{2}$-adrenergic agonists, including endogenous plasma catecholamines. Despite upregulation of AFC in pathologic conditions that may predispose to pulmonary edema, AFC is impaired in the majority of patients with ARDS $(17,38)$.

Initial studies were performed on passively rewarmed, nonperfused human lung lobes, in which AFC was $4 \%$ per hour (3). Using an intact ex vivo human lung preparation, Frank et al. (7) demonstrated that normothermic perfusion raised the rate of AFC from $4 \%$ to $20 \%$. Treatment with a $\beta$-agonist increased the AFC to $40 \%$, perhaps explaining the clinical observation that hydrostatic pulmonary edema in patients with congestive heart failure can be resolved rapidly when left atrial pressure is lowered with diuretics and afterload reduction. 
Table 1. Physiologic endpoints in the ex vivo-perfused human lung

\begin{tabular}{|c|c|c|c|}
\hline Experimental measure & Physiologic correlate & Expected range & References \\
\hline Lung weight gain & Endothelial permeability & $\begin{array}{c}25 \%-100 \% \text { in controls }{ }^{A} \\
100 \%-300 \% \text { in severe injury }\end{array}$ & 7,29 \\
\hline $\begin{array}{l}\text { Movement of Evans blue-labeled } \\
\text { albumin into airspaces }\end{array}$ & Endothelial and epithelial permeability & $\begin{array}{l}<10 \mu \mathrm{g} / \mathrm{ml} \text { in control lungs } \\
10-50 \mu \mathrm{g} / \mathrm{ml} \text { after injury }\end{array}$ & 29,30 \\
\hline Extravascular wet/dry ratio & Pulmonary edema & $\begin{array}{c}\text { Normal ratio }<4.2 \\
\text { Mild edema } 4.2-5.0 \\
\text { Moderate edema 5.1-6.0 } \\
\text { Severe edema }>6.0\end{array}$ & 4 \\
\hline Pulmonary arterial pressure & Right atrial pressure & Normal $<10$ mmHg & $31,41-43$ \\
\hline Alveolar fluid clearance & Alveolar fluid clearance & Normal $10 \%-50 \%$ & 34 \\
\hline $\mathrm{PaO}_{2}$ & Gas exchange & Normal $140-170 \mathrm{mmHg}$ & 30 \\
\hline Compliance and tracheal pressures & Lung compliance & $\begin{array}{l}\text { Normal lung compliance: } 15-20 \mathrm{ml} / \mathrm{cmH}_{2} \mathrm{O} \\
\text { Normal tracheal pressure: } 15-20 \mathrm{~cm} \mathrm{H}_{2} \mathrm{O}\end{array}$ & $30,32,42$ \\
\hline \multicolumn{4}{|c|}{$\begin{array}{l}\text { ATotal weight gain of lung with start weight prior to perfusion and end weight after } 6 \text { hours of perfusion in unselected lungs. Shaver et al. ( } 29 \text { ) excluded } \\
\text { lungs with significant weight gain over } 2 \text { hours and reported weight change of } 5 \%-25 \% \text { over } 2 \text { hours of perfusion in lungs injured with cell-free hemoglob } \\
{ }^{B} \text { Evans blue albumin in bronchoalveolar lavage after } 2 \text { hours of perfusion with or without injury ( } 29 \text { ). Park et al. ( } 30 \text { ) reported a ratio of approximately } 5: 1 \\
\text { of Evans blue accumulation in a lobe injured with E. coli, compared with a control lobe. 'Flow should be adjusted to keep pulmonary arterial pressure less } \\
\text { than } 10 \mathrm{mmHg} \text { to avoid hydrostatic edema. When used as an experimental outcome, pressures of } 0-5 \mathrm{mmHg} \text { have been routinely measured ( } 30 \text { ). }\end{array}$} \\
\hline
\end{tabular}

In the ex vivo-perfused human lung preparation, AFC is inversely related to lung weight gain (7) and depends on the presence of an intact epithelium (33) and measurement as follows. After a period of equilibration, a catheter (PE 240 tubing, BD Biosciences) is inserted through a side port in the endobronchial tube and advanced into the desired lobe until gentle resistance is met. A known volume (typically $100-150 \mathrm{ml}$ ) of $37^{\circ} \mathrm{C}$ $5 \%$ BSA in normal saline is instilled via the catheter into the distal airspaces. Fluid samples are obtained at 5 and 35 minutes, and the total protein in each sample is measured by refractometry. AFC is calculated using the following formula: $\operatorname{AFC}(\% / h)=2(1-\mathrm{Ci} / \mathrm{Cf})$, where $\mathrm{Ci}$ is the protein concentration of the 5 -minute sample and $\mathrm{Cf}$ is the protein concentration of the 35-minute sample. AFC may be measured in multiple lobes of the same lung or at multiple time points in the same lobe. For example, one group measured the AFC in a control lobe, then measured the AFC in a separate lobe following injury with E. coli (30). This method has been validated in several experimental preparations (38), and this approach has been adapted to measure the AFC rate in patients with hydrostatic (left heart failure) or increased permeability pulmonary edema (ARDS) by measuring the change in protein concentration of undiluted pulmonary edema fluid over time $(17,39,40)$.

Pulmonary vascular resistance. Pulmonary arterial pressures can be monitored continuously via a pulmonary arterial catheter in the circuit and pulmonary vascular resistance calculated. For example, one group reported that the addition of MSC-derived microvesicles to a human lung model of E. coli pneumonia associated with a trend toward a decrease in pulmonary arterial pressure and pulmonary vascular resistance (30). Another group of investigators perfused isolated human lungs or lobes from patients who underwent elective resection for cancer $(41,42)$. The authors demonstrated that addition of endothelin-1, endothelin-3, and hydrogen sulfide to the perfusate led to significant decreases in pulmonary vascular resistance, as measured by a drop in pulmonary arterial pressure. These changes correlated with arterial vasodilation, measured as a drop in isometric tension in isolated rings of human pulmonary artery. Of note, hydrogen sulfide also caused a significant reduction in bronchial airway pressures in ventilated lobes. The same group used an isolated perfused lobe to study whether rewarming after deep hypothermia in cardiothoracic surgery predisposes patients to acute postoperative pulmonary hypertension (43). This group reported that hypothermia blunted arterial responses to vasoconstriction but arterial responses normalized after warming, and there was no evidence of a hypothermic preconditioning effect.

Microbiology. The ex vivo-perfused human lung preparation is also valuable as a model of bacterial pneumonia and bacteremia. Several investigators have studied bacterial activity in the different compartments of the lung and bacterial movement between compartments $(30,31,44)$. For example, allogenic MSCs given intrabronchially or in the perfusate decreased total bacterial load in the alveolar fluid 6 hours after intrabronchial E. coli instillation, in part through enhanced bacterial phagocytosis by monocytes (31). Moreover, the addition of MSCs prevented translocation of $E$. coli from the airspaces to the perfusate. 
Table 2. Histologic, microbiologic, and immunologic studies in the ex vivo-perfused human lung

\begin{tabular}{|c|c|c|}
\hline Experimental measure & Examples & References \\
\hline Histology & E. coli and E. coli endotoxin tissue injuries & $21,30,31$ \\
\hline Microbiology & E. coli bacterial load in alveolar fluid and in perfusate & 30,31 \\
\hline Immune cells in airspaces & Neutrophil influx in alveolar fluid & 21, 30, 31 \\
\hline Cytokine secretion & IL-1 $\beta$, IL-8, TNF- $\alpha$ & $21,30,31,45$ \\
\hline $\begin{array}{l}\text { Epithelial or endothelial injury } \\
\text { biomarkers }\end{array}$ & RAGE, SP-D, vWF/Ag & 7, 33 \\
\hline
\end{tabular}

Treatment with MSC-derived microvesicles alone similarly decreased alveolar bacterial load and enhanced monocyte phagocytosis (30). Another group of investigators studied the differential effects of Streptococcus pneumoniae injected into the perfusate or instilled into the distal airspaces and found that bacteria introduced via either route produced endothelial injury, but only airspace infection was sufficient to produce epithelial injury (44). These studies have provided insight into the mechanisms of pulmonary defenses against pneumonia and bacteremia, for both Gram-positive and Gram-negative organisms, and have demonstrated several mechanisms by which MSCs exert antibacterial effects in the lung.

Histopathology. Injury of the ex vivo-perfused human lung preparation with intrabronchial endotoxin or E. coli leads to septal thickening and increased cellularity $(21,30,31)$. Instillation of MSCs or MSC-conditioned media reduced edema and cellularity following intrabronchial endotoxin injury, and MSC-conditioned media reduced the absolute neutrophil count in alveolar fluid following endotoxin injury (21). In subsequent work, this research group studied the phagocytic function of alveolar macrophages following injury of the lung with intrabronchial E. coli and treatment with intravenous or intrabronchial MSCs or the MSC paracrine product keratinocyte growth factor (31). Treatment with intrabronchial MSCs after E. coli pneumonia significantly increased the percentage of bacteria-containing alveolar macrophages (percentage of phagocytosis) and the average number of bacteria per macrophage (phagocytic index).

In a separate study, the histologic features of 29 pairs of lungs that had been rejected for transplantation were assessed (4); $62 \%$ had normal or mildly abnormal histologic findings. When histology was combined with AFC and microbiologic analysis, 12 pairs of lungs (41\%) were identified that might have been suitable for transplantation. These data have highlighted the need for prospective assessment of the donor selection process for lung transplantation.

\section{Ex vivo-perfused human lung: a therapeutic-testing platform}

The ex vivo-perfused human lung can be useful for testing novel therapeutics in acute lung injury and has laid the groundwork for some clinical trials in lung donor management (45) and cell-based therapy for the treatment of acute lung injury $(46,47)$. Examples of therapeutics tested in the ex vivo-perfused human lung are described in Table 3.

Recently, the system has been used to explore the potential roles of MSCs, MSC culture media, and MSC-derived microvesicles. In one study, human bone marrow-derived MSCs restored AFC in human lungs injured with intrabronchial E. coli LPS (21) or with live E. coli (31). Of note, the effect was observed after intrabronchial instillation of the MSCs $\left(5 \times 10^{6}\right.$ cells) as well as after intravenous infusion (in the perfusate). In addition to restoring a normal AFC rate, injured MSC-treated lungs had a decrease in inflammatory infiltrate and increased bacterial killing that could be explained in part by increased macrophage phagocytosis and secretion of antimicrobial factors. Subsequently, the safety of human MSCs in moderate to severe ARDS was demonstrated in phase I and IIa clinical trials $(48,49)$, and a phase IIb trial is in the planning stages. In other studies, the investigators found that intrabronchial instillation of MSC culture media or intravenous infusion of MSC-derived microvesicles has similar antimicrobial effects and similar beneficial effects on alveolar epithelial and endothelial function $(21,30)$.

Human MSCs and MSC-derived microvesicles also restore AFC and decrease weight gain in human lungs that have been rejected for transplantation $(28,32)$. These data suggest a potential method to expand the supply of donor lungs for transplantation. Similarly, another research group found that acetaminophen reduced cell-free hemoglobin-mediated oxidative injury in donor lungs rejected for transplant (29). Treated lungs had reduced endothelial permeability to protein and a decrease in weight gain. 
Table 3. Therapeutics tested in the ex vivo human lung

\begin{tabular}{|c|c|c|c|}
\hline Human mesenchymal stem cells & $\begin{array}{l}\text { E. coli endotoxin, live } E \text {. coli bacteria, } \\
\text { lungs rejected for transplantation }\end{array}$ & $\begin{array}{l}\text { AFC, extra lung water, endothelial permeability, } \\
\text { bacterial load, neutrophil count, phagocytic activity }\end{array}$ & $21,28,31$ \\
\hline MSC-derived microvesicles & $\begin{array}{l}\text { Live E. coli bacteria, lungs rejected for } \\
\text { transplantation }\end{array}$ & $\begin{array}{c}\text { AFC, lung weight gain, endothelial permeability, } \\
\text { bacterial load }\end{array}$ & 32 \\
\hline Recombinant KGF & E. coli endotoxin, live E. coli bacteria & $\begin{array}{l}\text { AFC, inflammatory cytokines bacterial load, } \\
\text { neutrophil count, phagocytic activity }\end{array}$ & 21, 31 \\
\hline $\begin{array}{l}\text { Acetaminophen } \\
\text { KGF, keratinocyte growth factor. }\end{array}$ & Intravascular cell-free hemoglobin & Lung weight gain, endothelial permeability & 29 \\
\hline
\end{tabular}

\section{Clinical applications of ex vivo lung perfusion}

In parallel with the translational research that our group and others have carried out with the single-lung ex vivo preparation described here, important progress has been made with commercially available 2-lung EVLP systems. These efforts have focused primarily on ex vivo conditioning or treatment of donor lungs before transplantation. For example, one group demonstrated that treatment of hepatitis $\mathrm{C}$ virus-infected lungs with phototherapy during EVLP decreased viral infectivity (50) and that treatment of human lungs rejected for transplant because of concerns of infection with broad-spectrum antibiotics during EVLP decreased bacterial counts in bronchoalveolar lavage (51). Several groups have demonstrated the feasibility of treating donor lungs with pulmonary emboli using urokinase or alteplase during EVLP before successful transplantation $(52,53)$. In addition, several groups have used EVLP systems to study the mechanisms of primary graft dysfunction (54) and to test novel therapeutics $(50,55)$.

\section{Limitations of the model}

The ex vivo lung preparation has several important limitations. There is significant heterogeneity in human lungs because of donor characteristics, such as sex, age, smoking history, and underlying diseases, and pre-procurement variables, such as trauma, ventilator-induced lung injury, and ventilator-associated pneumonia. It is straightforward to measure clearance of alveolar edema fluid from the airspaces and to follow fluid and proteins that are cleared by the pulmonary venous circulation; however, other important pathways, such as lymphatic drainage and drainage of fluid into the pleural space, cannot be measured (34). Similarly, the absence of other organs, such as the liver and spleen, facilitates direct study of pulmonary physiology; however, these tissues may be relevant to the study of pulmonary immune function and to the testing of novel therapeutics in the lung. Because the ex vivo lung preparation is not a closed system, gas exchange in the lung cannot be reliably measured. To date, most experiments have been limited to 6 to 10 hours, although the use of a bioreactor to extend studies up to 24 to 48 hours may be possible. It is also important to note that conducting these experiments requires a dedicated experimental setup and a research team with flexible hours because donor lung availability is unpredictable and consistent experimental methods are critical.

\section{Conclusions}

Acute and chronic respiratory diseases remain an important clinical problem. Historically, our understanding of the mechanisms of injury has been limited by the difficulty of reliably modeling lung injury in vitro or in animal models and by the challenges of isolating primary human cells from surgical samples. However, donor human lungs that have been rejected for transplant have proved a valuable resource for clinically relevant data, an important source of human pulmonary cells, and a new translational platform to test mechanisms of injury and new therapeutics.

\section{Acknowledgments}

JTR is supported by NIH T32DK007573-23. NN is supported by an international research grant from the Société Française d'Anesthésie-Réanimation (Paris, France), a research grant from the Association Chirurgicale pour le Développement et l'Amélioration des Techniques de Dépistage de Traitement des Mala- 
dies Cardiovasculaires (Paris, France), and a grant from the Journées Rennaises d'Anesthésie-Réanimation association (Rennes, France). JWL is supported in part by the National Heart, Lung, and Blood Institute grant HL113022. LBW is supported by NIH grants HL135849 and HL126176. MAM is supported in part by NIH grants HL126456, HL126176, HL140026, and HL134828. This research was supported in part by the UCSF Nina Ireland Program for Lung Health. The authors thank Diana Lim for her work in preparing Figure 2. The authors thank donor families for their gift to research and Donor Network West and Tennessee Donor Services for their assistance in obtaining organs for research.

Address correspondence to: Michael A. Matthay, 505 Parnassus Ave., M-917, UCSF, San Francisco, California 94143, USA. Phone: 415.353.1206; Email: Michael.Matthay@ucsf.edu.

1. Ware LB. Modeling human lung disease in animals. Am J Physiol Lung Cell Mol Physiol. 2008;294(2):L149-L150.

2. Matthay MA, et al. Acute respiratory distress syndrome. Nat Rev Dis Primers. 2019;5(1):18.

3. Sakuma T, Okaniwa G, Nakada T, Nishimura T, Fujimura S, Matthay MA. Alveolar fluid clearance in the resected human lung Am J Respir Crit Care Med. 1994;150(2):305-310.

4. Ware LB, et al. Assessment of lungs rejected for transplantation and implications for donor selection. Lancet. 2002;360(9333):619-620.

5. Cypel M, et al. Normothermic ex vivo lung perfusion in clinical lung transplantation. NEngl J Med. 2011;364(15):1431-1440.

6. Fisher A, et al. An observational study of Donor Ex Vivo Lung Perfusion in UK lung transplantation: DEVELOP-UK. Health Technol Assess. 2016;20(85):1-276.

7. Frank JA, Briot R, Lee JW, Ishizaka A, Uchida T, Matthay MA. Physiological and biochemical markers of alveolar epithelial barrier dysfunction in perfused human lungs. Am J Physiol Lung Cell Mol Physiol. 2007;293(1):L52-L59.

8. Valapour M, et al. OPTN/SRTR 2016 Annual Data Report: Lung. Am J Transplant. 2018;18(suppl 1):363-433.

9. Ware LB, et al. Comparison of chest radiograph scoring to lung weight as a quantitative index of pulmonary edema in organ donors. Clin Transplant. 2012;26(5):665-671.

10. Warren MA, et al. Severity scoring of lung oedema on the chest radiograph is associated with clinical outcomes in ARDS. Thorax. 2018;73(9):840-846

11. Ware LB, et al. Donor smoking is associated with pulmonary edema, inflammation and epithelial dysfunction in ex vivo human donor lungs. Am J Transplant. 2014;14(10):2295-2302.

12. Ware LB, Golden JA, Finkbeiner WE, Matthay MA. Alveolar epithelial fluid transport capacity in reperfusion lung injury after lung transplantation. Am J Respir Crit Care Med. 1999;159(3):980-988.

13. Fang X, Matthay MA. Measurement of protein permeability and fluid transport of human alveolar epithelial type II cells under pathological conditions. Methods Mol Biol. 2018;1809:121-128.

14. Kosmider B, Mason RJ, Bahmed K. Isolation and characterization of human alveolar type II cells. Methods Mol Biol. 2018;1809:83-90.

15. Fang X, et al. Contribution of CFTR to apical-basolateral fluid transport in cultured human alveolar epithelial type II cells. Am J Physiol Lung Cell Mol Physiol. 2006;290(2):L242-L249.

16. Agrawal PB, et al. The epithelial sodium channel is a modifier of the long-term nonprogressive phenotype associated with F508del CFTR mutations. Am J Respir Cell Mol Biol. 2017;57(6):711-720.

17. Ware LB, Matthay MA. Alveolar fluid clearance is impaired in the majority of patients with acute lung injury and the acute respiratory distress syndrome. Am J Respir Crit Care Med. 2001;163(6):1376-1383.

18. Lee JW, et al. Acute lung injury edema fluid decreases net fluid transport across human alveolar epithelial type II cells. $J$ Biol Chem. 2007;282(33):24109-24119.

19. Fang X, Neyrinck AP, Matthay MA, Lee JW. Allogeneic human mesenchymal stem cells restore epithelial protein permeability in cultured human alveolar type II cells by secretion of angiopoietin-1. J Biol Chem. 2010;285(34):26211-26222.

20. Fang X, et al. Human mesenchymal stem (stromal) cells promote the resolution of acute lung injury in part through lipoxin A4. J Immunol. 2015;195(3):875-881.

21. Lee JW, Fang X, Gupta N, Serikov V, Matthay MA. Allogeneic human mesenchymal stem cells for treatment of E. coli endotoxin-induced acute lung injury in the ex vivo perfused human lung. Proc Natl Acad Sci USA. 2009;106(38):16357-16362.

22. Schwede M, et al. Effects of bone marrow-derived mesenchymal stromal cells on gene expression in human alveolar type II cells exposed to TNF- $\alpha$, IL-1 $\beta$, and IFN- $\gamma$. Physiol Rep. 2018;6(16):e13831.

23. Wang $\mathrm{C}$, et al. Expansion of hedgehog disrupts mesenchymal identity and induces emphysema phenotype. J Clin Invest. 2018;128(10):4343-4358.

24. Ning W, et al. Cigarette smoke stimulates matrix metalloproteinase-2 activity via EGR-1 in human lung fibroblasts. Am J Respir Cell Mol Biol. 2007;36(4):480-490.

25. Li CJ, Ning W, Matthay MA, Feghali-Bostwick CA, Choi AM. MAPK pathway mediates EGR-1-HSP70-dependent cigarette smoke-induced chemokine production. Am J Physiol Lung Cell Mol Physiol. 2007;292(5):L1297-L1303.

26. Desch AN, et al. Flow cytometric analysis of mononuclear phagocytes in nondiseased human lung and lung-draining lymph nodes. Am J Respir Crit Care Med. 2016;193(6):614-626.

27. Comhair SA, Xu W, Mavrakis L, Aldred MA, Asosingh K, Erzurum SC. Human primary lung endothelial cells in culture. Am J Respir Cell Mol Biol. 2012;46(6):723-730.

28. McAuley DF, et al. Clinical grade allogeneic human mesenchymal stem cells restore alveolar fluid clearance in human lungs rejected for transplantation. Am J Physiol Lung Cell Mol Physiol. 2014;306(9):L809-L815.

29. Shaver CM, et al. Cell-free hemoglobin promotes primary graft dysfunction through oxidative lung endothelial injury. JCI 
Insight. 2018;3(2):98546.

30. Park J, et al. Therapeutic effects of human mesenchymal stem cell microvesicles in an ex vivo perfused human lung injured with severe. Thorax. 2019;74(1):43-50.

31. Lee JW, Krasnodembskaya A, McKenna DH, Song Y, Abbott J, Matthay MA. Therapeutic effects of human mesenchymal stem cells in ex vivo human lungs injured with live bacteria. Am J Respir Crit Care Med. 2013;187(7):751-760.

32. Gennai S, Monsel A, Hao Q, Park J, Matthay MA, Lee JW. Microvesicles derived from human mesenchymal stem cells restore alveolar fluid clearance in human lungs rejected for transplantation. Am J Transplant. 2015;15(9):2404-2412.

33. Briot R, Frank JA, Uchida T, Lee JW, Calfee CS, Matthay MA. Elevated levels of the receptor for advanced glycation end products, a marker of alveolar epithelial type I cell injury, predict impaired alveolar fluid clearance in isolated perfused human lungs Chest. 2009;135(2):269-275.

34. Matthay MA, Folkesson HG, Clerici C. Lung epithelial fluid transport and the resolution of pulmonary edema. Physiol Rev. 2002;82(3):569-600

35. Christie JD, et al. Primary graft failure following lung transplantation. Chest. 1998;114(1):51-60.

36. Wiener-Kronish JP, Broaddus VC, Albertine KH, Gropper MA, Matthay MA, Staub NC. Relationship of pleural effusions to increased permeability pulmonary edema in anesthetized sheep. J Clin Invest. 1988;82(4):1422-1429.

37. Staub NC. Pulmonary edema. Physiol Rev. 1974;54(3):678-811.

38. Matthay MA. Resolution of pulmonary edema. Thirty years of progress. Am J Respir Crit Care Med. 2014;189(11):1301-1308.

39. Zeyed YF, Bastarache JA, Matthay MA, Ware LB. The severity of shock is associated with impaired rates of net alveolar fluid clearance in clinical acute lung injury. Am J Physiol Lung Cell Mol Physiol. 2012;303(6):L550-L555.

40. Ware LB, Fremont RD, Bastarache JA, Calfee CS, Matthay MA. Determining the aetiology of pulmonary oedema by the oedema fluid-to-plasma protein ratio. Eur Respir J. 2010;35(2):331-337.

41. Bennett RT, Jones RD, Morice AH, Smith CF, Cowen ME. Vasoconstrictive effects of endothelin-1, endothelin-3, and urotensin II in isolated perfused human lungs and isolated human pulmonary arteries. Thorax. 2004;59(5):401-407.

42. Ariyaratnam P, Loubani M, Morice AH. Hydrogen sulphide vasodilates human pulmonary arteries: a possible role in pulmonary hypertension? Microvasc Res. 2013;90:135-137.

43. Ariyaratnam P, et al. The effect of deep hypothermia on the human pulmonary circulation. J Therm Biol. 2014;40:20-24

44. Ross JT, Nesseler N, Minus E, Gotts JE, Matthay MA. Differential effects of high dose bacteria on lung endothelium and alveolar epithelium in the ex vivo perfused human lung. J Surg Res. In press.

45. Ware LB, et al. A randomized trial of the effects of nebulized albuterol on pulmonary edema in brain-dead organ donors. Am J Transplant. 2014;14(3):621-628.

46. McAuley DF, et al. Keratinocyte growth factor for the treatment of the acute respiratory distress syndrome (KARE): a randomised, double-blind, placebo-controlled phase 2 trial. Lancet Respir Med. 2017;5(6):484-491.

47. Perkins GD, et al. The beta agonist lung injury trial prevention. A randomized controlled trial. Am J Respir Crit Care Med. 2014;189(6):674-683.

48. Wilson JG, et al. Mesenchymal stem (stromal) cells for treatment of ARDS: a phase 1 clinical trial. Lancet Respir Med. 2015;3(1):24-32.

49. Matthay MA, et al. Treatment with allogeneic mesenchymal stromal cells for moderate to severe acute respiratory distress syndrome (START study): a randomised phase 2a safety trial. Lancet Respir Med. 2019;7(2):154-162.

50. Galasso $\mathrm{M}$, et al. Inactivating hepatitis $\mathrm{C}$ virus in donor lungs using light therapies during normothermic ex vivo lung perfusion. Nat Commun. 2019;10(1):481.

51. Nakajima D, et al. Ex vivo perfusion treatment of infection in human donor lungs. Am J Transplant. 2016;16(4):1229-1237.

52. Machuca TN, et al. Injury-specific ex vivo treatment of the donor lung: pulmonary thrombolysis followed by successful lung transplantation. Am J Respir Crit Care Med. 2013;188(7):878-880.

53. Inci I, Yamada Y, Hillinger S, Jungraithmayr W, Trinkwitz M, Weder W. Successful lung transplantation after donor lung reconditioning with urokinase in ex vivo lung perfusion system. Ann Thorac Surg. 2014;98(5):1837-1838.

54. Caldarone L, et al. Neutrophil extracellular traps in human ex vivo lung perfusion as a biomarker of lung injury. J Heart Lung Transplant. 2018;37(4):S33

55. Weathington NM, et al. Ex vivo lung perfusion as a human platform for preclinical small molecule testing. JCI Insight. 2018;3(19):95515 